\title{
EVALUACIÓN DEL CAPITAL CULTURAL EN LA FRONTERA URUGUAYO BRASILERA
}

\author{
IGNACIO PABLO TRAVERSA TEJERO \\ SISTEMA NACIONAL DE INVESTIGADORES \\ URUGUAI \\ IGTRAVERSA@GMAIL.COM
}

JUAN FRANCISCO XAVIER ALVEZ CENTRO REGIONAL DE PROFESORES DEL NORTE URUGUAI JFXAVIERALVEZ@GMAIL.COM

JORGE RODRÍGUEZ MONTEBLANCO CENTRO REGIONAL DE PROFESORES DEL NORTE URUGUAI JRMONTEBLANCO@GMAIL.COM

HTTP://DX.DOI.ORG/10.5902/2316882X25961 


\section{AVALIAÇÃO DO CAPITAL CULTURAL NA FRONTEIRA BRASILEIRA DO URUGUAI}

Resumo: Este estudo foi realizado em um espaço de interface com menor desenvolvimento humano. Foram coletados dados educacionais em dois países: Uruguai e Brasil e calculado o coeficiente de determinação de Pearson (R). Gerou se uma metodologia válida e conclui se que a escolaridade média dos pais explica em 0,33 a escolaridade dos filhos. A influência dos pais explica pelos menos um terço os anos de esolarização futura de um indivíduo tomado ao acaso, com um efeito maior do pai $(0,37)$ que da mãe $(0,27)$.

Palavras-chave: desigualdade, educação, ensino, ambiente familiar.

\section{EVALUACIÓN DEL CAPITAL CULTURAL EN LA FRONTERA URU- GUAYO BRASILERA}

El presente estudio fue realizado en un espacio de interface con menor desarrollo humano. Fueron recabados datos educativos en dos países: Uruguay y Brasil. Se calculó el coeficiente de determinación de Pearson $(R)$, se llegó a una metodología válida y se concluye que la escolarización promedio de los padres explica en 0,33 la escolarización de los hijos. La influencia parental explica por lo menos en un tercio los años de escolarización futura de un individuo tomado al azar, con un mayor efecto del padre $(0,37)$ sobre la madre $(0,27)$.

Palabras clave: desigualdad, educación, escolarización, entorno familiar.

\section{ASSESSMENT OF CULTURAL CAPITAL IN THE BRAZILIAN BORDER OF URUGUAY}

The present study was carried out in an interface space with less human development. Educational data were collected in two countries: Uruguay and Brazil. The Pearson ( $R$ ) coefficient of determination was calculated, a valid methodology was reached and it was concluded that the average schooling of the parents explains the schooling of the children by 0.33 . Parental influence accounts for at least one third of the years of future schooling of a random individual, with a greater effect of the father (0.37) on the mother (0.27).

Keywords: inequality, education, schooling, family environment. 


\section{INTRODUCCIÓN}

Uruguay y Brasil tienen una frontera de más 1000 km de extensión que se encuentra exenta de elementos geográficos relevantes que impidan la libre circulación de la población entre ambos países. Es un territorio de tensión y de encuentro, en donde es factible lograr una cooperación horizontal a nivel de las denominadas ciudades gemelas. Avanzar en el proceso de integración regional supone superar la visión de la frontera como el último confín, para ser contemplada humana, económica y socialmente como espacio de oportunidades vinculado a estrategias de desarrollo y un nuevo sentido de soberanía (RÓTULO; DAMIANI, 2010). Una forma de potenciar la integración interestatal y ciudadana es lo que se conoce como "integración fronteriza", que es un proceso de integración amplio, en donde se amplifican y evidencian las dificultades mayores de la integración regional, y donde son necesarios acciones de complementación de recursos humanos. Oliveros (2002) entiende por integración fronteriza al proceso convenido por dos estados de los territorios fronterizos colindantes a través de acuerdos o tratados para el desarrollo. Los departamentos uruguayos fronterizos con Brasil totalizan tienen una población de 329.657 habitantes contenidos en una superficie de $45.497 \mathrm{~km}^{2}$, mientras que los municipios brasileños de frontera con Uruguay totalizan 38.343 $\mathrm{km}^{2}$ y 468.821 habitantes (MAZZEI; DE SOUZA, 2012).

Históricamente, en esta faja de frontera el español no logró excluir a la lengua portuguesa quedando yuxtapuesta con los denominados dialectos portugueses en el Uruguay (DPU). La presencia humana determina el paisaje cultural, que es la resultante de la interacción estrecha entre los seres humanos y el ambiente natural a lo largo de un proceso dilatado de varios siglos, la región hoy es el producto del pasaje de nativos, portugueses, españoles, afro descendientes y emigrantes diversos (TRAVERSA, 2015). El espacio social es construido de tal modo que los agentes o los grupos son distribuidos en él en función de su posición en las distribuciones estadísticas según los dos principios de diferenciación, el capital económico y el capital cultural (BOURDIEU, 2010).

Hannerz (2010) nos hace percibir que si las fronteras no son naturales (como es el caso de Uruguay-Brasil en gran parte de la extensión), se convierten en lo que las personas hacen de ellas. Hay quienes otorgan más importancia a las fronteras, y quienes menos, hay quienes centran la aten-

Rev.Cad.Comun. Santa Maria, v.21, n.3, art 6, p. 105 de 123, set/dez.2017 
ción sobre la diferencia y la discontinuidad, y hay quienes no. Existe una marcada tendencia a una distribución urbana de la población en ambos estados fronterizos; para todo Uruguay del 94,6 \% (2011) y para el Estado de Rio Grande do Sul del 85,0 \% (2010). Es posible afirmar que a todos los niveles espaciales observados, tanto a nivel nacional, estadual, departamentos uruguayos y de los municipios brasileños a la vez que se confirma una fuerte primacía urbana, ese rasgo de urbanización es más acentuado entre las áreas uruguayas respecto a las áreas brasileñas abordadas (MAZZEI; DE SOUZA, 2012).

La región uruguaya fronteriza con Brasil, experimentó importantes cambios durante los años noventa, debido a procesos de diversificación económica, de un alto nivel agroexportador. No obstante, las desigualdades internas y los niveles de carencias y pobreza continúan siendo muy significativos (VEIGA; RIVOIR, 2004). Si bien ya desde la década de 1970 comenzaron a vislumbrarse transformaciones en la dinámica de la vida conyugal, puede decirse que el segundo quinquenio de la década de los ochenta fue el escenario de un giro sin precedentes en la historia de la familia uruguaya del siglo XX. En pocos años los casamientos descendieron a la mitad, los divorcios se duplicaron y las uniones libres comenzaron a ser una alternativa cada vez más frecuente frente al matrimonio legalizado. La combinación de estos procesos con las tendencias demográficas, sociales y económicas ha dado lugar a la transformación de la fisonomía de las familias uruguayas (CABELLA, 2007). De acuerdo con Veiga y Rivoir (2004) la fragmentación socio-territorial, no es una simple consecuencia de las desigualdades sociales, sino que es el resultado de la diferenciación social en el espacio. En tal sentido, constituyen procesos mutuamente retroalimentados, en la medida que la posición y la identidad de un área, está condicionada por su estructura socioeconómica. Así las diversas formas de segregación residencial y educativa, son manifestaciones de la distribución de las clases sociales en las ciudades y territorio.

El Índice de Desarrollo Humano (IDH) es una medida no ponderada que clasifica a los países y regiones en una escala 0-1 y permite considerar tres niveles de desarrollo humano: alto $(>0,800)$, medio $(0,500-0,799)$ y bajo $(<0,500)$ (UNNE, 2017). Es un indicador sintético de los logros medios obtenidos en las dimensiones fundamentales del desarrollo humano: vida larga y saludable, adquisición de conocimientos y disfrute de un nivel de vida digno. La dimensión educación se mide por los años promedio

Rev.Cad.Comun. Santa Maria, v.21, n.3, art 6, p. 106 de 123, set/dez.2017 
de escolaridad de los adultos de 25 años o más y por los años esperados de escolaridad de los niños en edad escolar (PNUD, 2017). La ciudad de Montevideo (metrópoli centralizadora de Uruguay) tiene un IDH de 0,841, superior a cualquiera de los departamentos fronterizos con Brasil (PNUD, 2016). De manera muy similar, la metrópoli y capital estatal de Rio Grande do Sul, Porto Alegre, tiene un IDH de 0,805, cifra superior a todos los municipios fronterizos con Uruguay (PNUD, 2010).

En un proceso de intención sostenida de llegar a la universalización de la enseñanza primaria y de la enseñanza secundaria, se ha llegado a que la enseñanza universitaria pública ya es una realidad en esta frontera uruguayo brasilera. De acuerdo con Fernández Aguerre, (2002), la descentralización se impulsó a fines de los años ochenta para mejorar la calidad de la educación y la disminuir las desigualdades de resultados. Para ello, los sistemas educativos apuntaron a objetivos de: atender las necesidades locales, contextualizar el currículum; disminuir la burocracia; impulsar innovaciones educativas, abrir espacios de participación, incrementar el compromiso financiero familiar y empresarial entre otros. En la búsqueda de una sociedad más justa Tenti Fanfani (2008) menciona que es necesaria una educación de calidad y una opción política basada en una distribución más equitativa del ingreso, que considere a la educación como un factor de fundamental importancia para el desarrollo económico. Es por ello que, el programa nacional de educación brasilero, estableció metas específicas de proveer ofertas de educación superior para el final de la década y ampliar la oferta de enseñanza primaria a la promoción del principio de gestión democrática de la educación pública (BRASIL, 2014).

Por lo expuesto, el enfoque metodológico basado en el estudio del espacio fronterizo y no de países queda justificado. Además y de acuerdo con Tenti Fanfani (2011) es preciso reconocer que si uno quiere intervenir con éxito en la realidad social, es mejor conocerla y comprenderla, una condición necesaria para la intervención y la política social. Fue por ello, que se analizó parte del territorio binacional (Uruguay-Brasil) a partir de un enfoque integrador con el objetivo de conocer el nivel educativo de la población fronteriza medido como años de escolarización. Como objetivos específicos: analizar el IDH de la región fronteriza entre ambos países y determinar la influencia del nivel educativo promedio de los padres en relación al nivel educativo de los hijos y proporcionar una metodología de abordaje que pueda ser reproducida en otros puntos de contactos de la vasta frontera.

Rev.Cad.Comun. Santa Maria, v.21, n.3, art 6, p. 107 de 123, set/dez.2017 


\section{METOdOLOGÍA}

\section{Área de estudio.}

La frontera uruguayo brasilera incluye los siguientes municipios uruguayos: Artigas, Rivera, Cerro Largo, Treinta y Tres y Rocha. Por su parte, los municipios brasileros son: Barra do Quaraí, Uruguaiana, Quaraí, Sant'Ana do Livramento, Dom Pedrito, Bagé, Aceguá, Erval, Jaguarão, Santa Vitória do Palmar y Chuí. La faja fronteriza se extiende en la dirección sureste-noroeste. El centro de dicha faja tiene cierta representatividad del todo por amortiguar los efectos extremos, en él se encuentran los municipios que fueron estudiados: Rivera 9,370 km², Sant'Ana do Livramento 6,950 km² y Dom Pedrito 5,192 $\mathrm{km}^{2}$. Debido a la alta concentración urbana dentro de estos municipios las ciudades capitales fueron la zona de colecta de datos de la investigación. A modo de ejemplo, el departamento de Rivera concentra un 31\% del total de la población de los cuatro departamentos uruguayos fronterizos (MAZZEI; DE SOUZA, 2012).

La población uruguaya fronteriza ha disminuido en los últimos años, la serie histórica ha sido la siguiente: 375.835 personas (1996), 375.645 personas (2004) y 374.467 personas (2011) (INE, 2017). Por el lado brasilero, el fenómeno de despoblamiento o decrecimiento es similar, el censo del año 2000 estimó un total de 481.899 personas y el censo del año 2010, 468.821 personas IBGE (2010). Uruguay ocupa la posición 54 del ranking del IDH y Brasil la posición 79 (tabla 1).

\begin{tabular}{|l|c|c|c|c|c|c|c|c|}
\hline País & 1990 & 2000 & 2010 & 2011 & 2012 & 2013 & 2014 & 2015 \\
\hline Uruguay & 0,692 & 0,742 & 0,780 & 0,784 & 0,788 & 0,791 & 0,794 & 0,795 \\
\hline Brasil & 0,611 & 0,685 & 0,724 & 0,730 & 0,734 & 0,747 & 0,754 & 0,754 \\
\hline
\end{tabular}

Tabla 1. Tendencia de la serie temporal del Índice de Desarrollo Humano (IDH) por país.

Editado de (UNDP, 2016)

\section{Colecta de datos.}

El estudio fue divido en dos etapas la primera referente a búsqueda bibliográfica y la segunda relativa a la colecta de datos de campo con análisis descriptivo de los datos levantados (FALCO et al., 2015). La búsqueda bibliográfica fue relativa a factores históricos y sociales de la población fronteriza y valores oficiales de de IDH en la región y a fenómenos migratorios y de distribución de la población. 
A los efectos de colectar los datos de campo, la población local de la faja fronteriza fue dividida en tres bloques: población de ingreso potencial a la universidad, población circulante y población comerciante. La primera constituida por jóvenes que están en cursos preparatorios para ingresar a la universidad, la segunda formada por personas que circulaban por los diferentes barrios de las tres ciudades y la tercera integrada por personas que trabajan en diferentes comercios de las ciudades mencionadas. En todos los casos las personas fueron entrevistadas de manera aleatoria.

En cada uno de los tres bloques fueron aplicados cuestionarios mixtos (preguntas abiertas y cerradas). Se diseñó y aplicó una encuesta (VALDÉS et. al., 2009), a manera de entrevista informal consistente en una conversación oral entre dos personas (entrevistado y entrevistador) (ROESCH, 2009; MARCONI, LAKATOS; 2011). Las variables recogidas fueron el grado de escolarización medido como número de años cursados de primaria, secundaria o enseñanza superior, tanto del entrevistado como de ambos padres.

Treinta o mas observaciones son consideradas muestras grandes (INFANTE, ZÁRATE DE LARA; 1994) y por el Teorema Central del Limite ese tamaño ya garantiza una distribución mínima confiable (MOURÃO-JUNIOR, 2009), pues la teoría establece que el valor medio observado en $n$ ensayos converge a $\mathrm{E}(\mathrm{X})$ (esperanza matemática) cuando $\mathrm{n}$ ? $\infty$ (INFANTE, ZÁRATE DE LARA; 1994). Fueron tomadas series de treinta observaciones hasta lograr la estabilización de la frecuencia relativa de la escolarización en años. Al comienzo existieron mayores oscilaciones pero en la medida que la muestra aumentó se llegó a la estabilización (figura 1) de la escolarización promedio de las madres y padres de los entrevistados. Cuando se alcanzó un error relativo acumulado (\%) de 0,13 y se habían aplicado 555 cuestionarios se interrumpió la colecta de datos, entendiéndose que la muestra brindaba suficiente información con un mínimo error. 


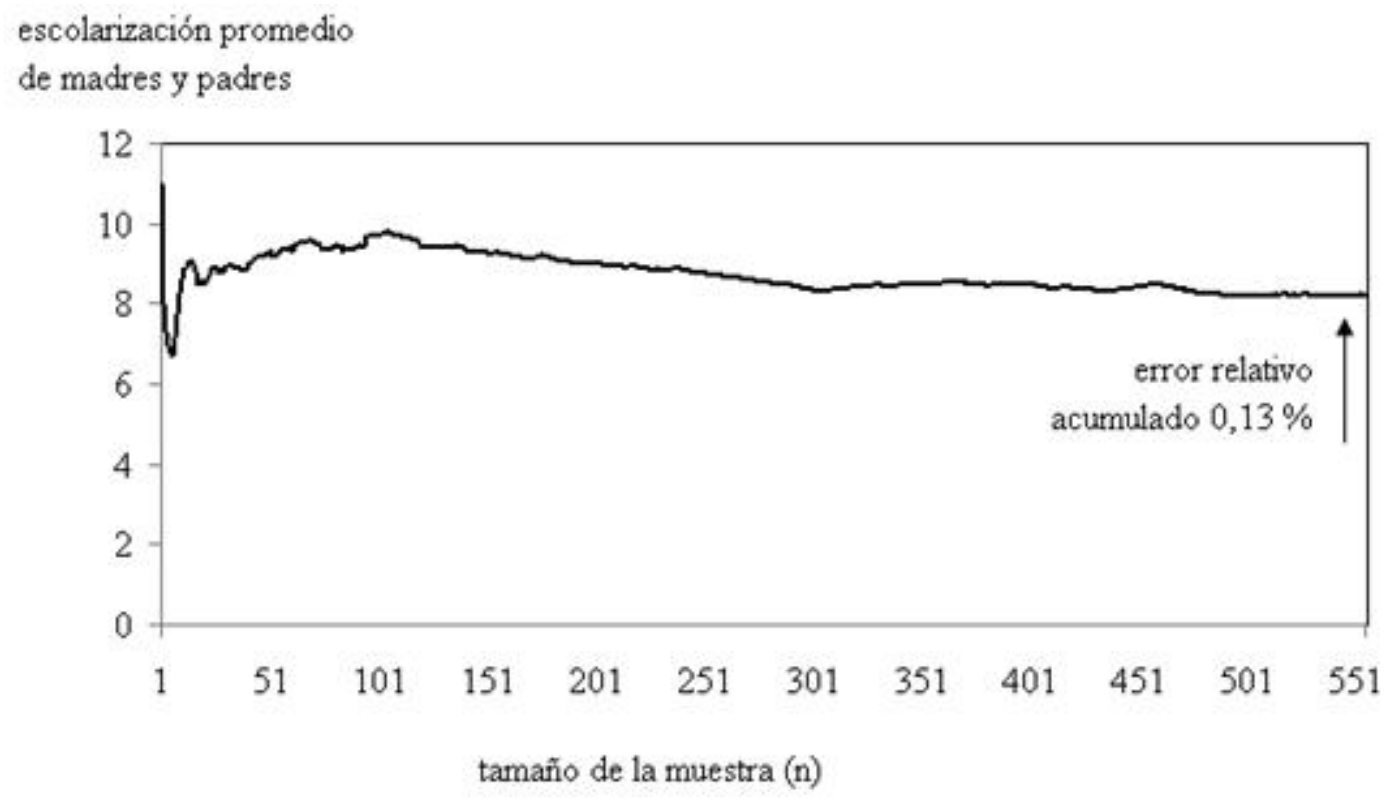

Figura 1. Estabilización de la escolarización del promedio de los padres de los entrevistados en la frontera uruguayo-brasilera.

\section{Procesamiento de datos.}

Los datos recabados fueron procesados por medio de funciones estadísticas de la planilla de cálculo Microsoft Excel, fueron aplicados métodos de análisis numéricos (parámetros de tendencia central y de dispersión), métodos tabulares y métodos gráficos (INFANTE, ZÁRATE DE LARA; 1994). De manera complementar se construyeron funciones que asociaron la escolarización promedio de los progenitores con la escolarización de los hijos. En este caso se utilizaron los datos referentes a personas que tenían 25 o más años de edad. En este estudio conjunto de variables, se calcularon el coeficiente de correlación ( $r$ ) y el coeficiente de determinación de Pearson (R). A los efectos de tener una visión integrada y una mejor interpretación, el trabajo cuantitativo fue complementado con un análisis cualitativo a los de los resultados obtenidos.

\section{RESULTADOS Y DISCUSIÓN}

En función del campo analizado el capital se presenta en la forma cultural, económica y social (BOURDIEU, 2000). Capital es todo aquello que puede entrar en las "apuestas" de los actores sociales, que es un "instrumento de apropiación de las oportunidades teóricamente ofrecidas a todos", o toda "energía social" susceptible de producir efectos en la compe-

Rev.Cad.Comun. Santa Maria, v.21, n.3, art 6, p. 110 de 123, set/dez.2017 
tencia social (BOURDIEU, 1993), una forma de poder, siempre usada para realizar los intereses de unos actores concretos, en tanto que capacidad para ejercer control (CALHOUN, 1993), o como "relación social que define la apropiación desigual de recursos" (MARTíN; IZQUIERDO, 1992) que está en este caso asociada a una región de frontera que tiene menor desarrollo humano y se encuentra alejada del centralismo capitalino. La distribución de la población del área fronteriza, muestra un acentuado grado de urbanización, así en 2011 el conjunto de los departamentos uruguayos fronterizos tienen un $93,6 \%$ de población urbana y el total de los municipios brasileños en 2010 tienen un $88,3 \%$ de población urbana (MAZZEI; DE SOUZA, 2012).

La forma institucionalizada del capital cultural está vinculada con los títulos académicos obtenidos por nivel educativo, siendo la educación una acumulación de transmisión cultural familiar y de transmisión cultural escolar (BOURDIEU, 1988). En números aproximados se encontró que solamente el $6 \%$ de la población entrevistada tiene los tres niveles educativos: superior, medio y primario; el 60\% tiene enseñanza media y básica, y un tercio tan sólo la enseñanza primaria o parte de ella (figura 2). En Uruguay ANEP (2000), encontró que mientras en las escuelas clasificadas como de contexto muy favorable dos tercios de las madres completaron la educación primaria, en las clasificadas como de contexto muy desfavorable ello ocurre apenas con una de cada catorce madres. Asimismo, la tasa de hacinamiento en los hogares es diez veces superior en estas últimas escuelas en relación a las primeras.

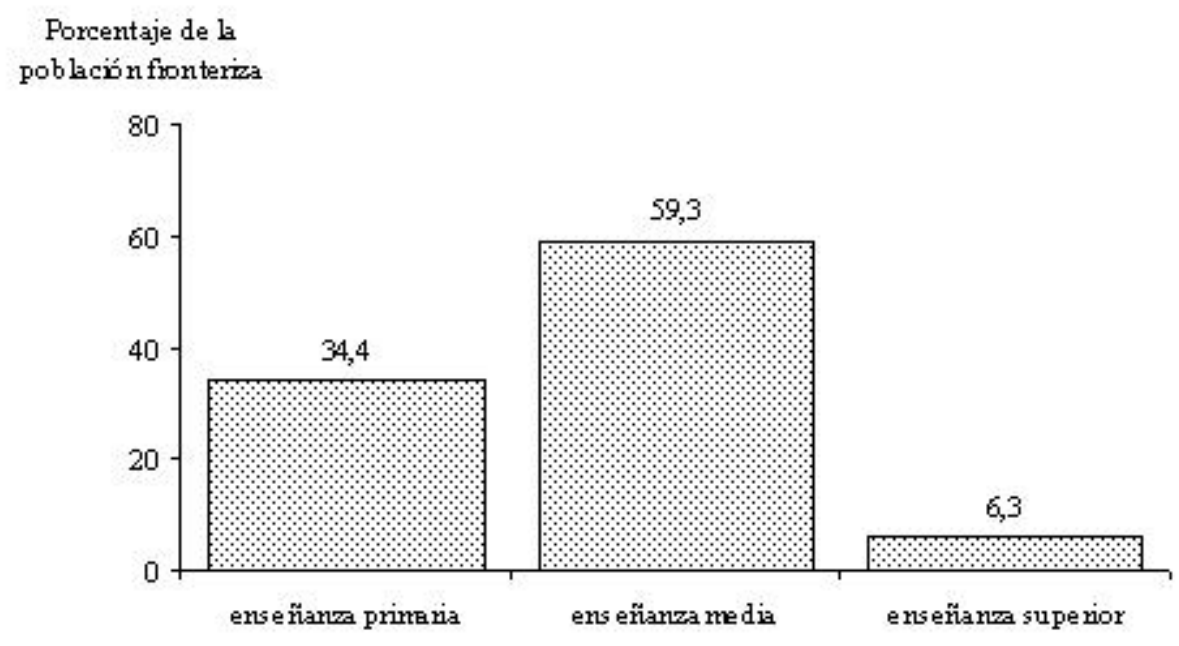

Figura 2. Niveles de educación (primaria-secundaria-superior) de los entrevistados en la frontera uruguayo-brasilera

Rev.Cad.Comun. Santa Maria, v.21, n.3, art 6, p. 111 de 123, set/dez.2017 
La escolarización media del entrevistado promedio en número de años es de 11, esto equivale a la enseñanza primaria completa, más 3-5 años de enseñanza secundaria dependiendo del país (Uruguay o Brasil), la mediana y la moda reflejan el mismo valor lo que indica una distribución simétrica entorno de la media. A la vez que el analfabetismo en Uruguay es menor que en el Estado de Rio Grande do Sul, el analfabetismo en el conjunto de los departamentos uruguayos de frontera es predominantemente menor respecto al analfabetismo en el conjunto de los municipios fronterizos brasileños (MAZZEI; DE SOUZA, 2012). No obstante lo anterior, los valores oficiales de IDH de los municipios fronterizos son similares (tabla 2).

\begin{tabular}{|c|c|c|}
\hline Municipio & País & IDH \\
\hline Artigas & Uruguay & 0,738 \\
\hline Rivera & Uruguay & 0,710 \\
\hline Cerro Largo & Uruguay & 0,740 \\
\hline Treinta y Tres & Uruguay & 0,741 \\
\hline Rocha & Uruguay & 0,756 \\
\hline Barra do Quarai & Brasil & 0,662 \\
\hline Uruguaiana & Brasil & 0,707 \\
\hline Quarai & Brasil & 0,704 \\
\hline Sant'Ana do Livramento & Brasil & 0,727 \\
\hline Dom Pedrito & Brasil & 0,708 \\
\hline Bagé & Brasil & 0,740 \\
\hline Aceguá & Brasil & 0,687 \\
\hline Erval & Brasil & 0,687 \\
\hline Jaguarão & Brasil & 0,707 \\
\hline Santa Vitória do Palmar & Brasil & 0,712 \\
\hline Chuí & Brasil & 0,706 \\
\hline
\end{tabular}

Tabla 2. Índice de Desarrollo Humano (IDH) por municipio fronterizo. Editado de (PNUD, 2010)

El promedio para los dieciséis municipios $(0,7145)$ pertenece a la categoría media, siendo la amplitud (diferencia entre el valor máximo y el mínimo) de apenas 0,094 (Rocha y Barra do Quarai) (tabla 3). 


\begin{tabular}{|c|c|}
\hline Estadístico & Valor \\
\hline Media & 0,715 \\
\hline Error típico & 0,006 \\
\hline Mediana & 0,709 \\
\hline Moda & 0,740 \\
\hline Desviación estándar & 0,025 \\
\hline Curtosis & $-0,054$ \\
\hline Coeficiente de asimetría & $-0,262$ \\
\hline Amplitud & 0,094 \\
\hline Mínimo & 0,662 \\
\hline Máximo & 0,756 \\
\hline Nivel de confianza (95,0\%) & 0,013 \\
\hline
\end{tabular}

Tabla 3. Valores estadísticos medios de la región fronteriza calculados a partir del IDH.

Es posible decir que la población parental (madres, padres, y promedio de padres) de los entrevistados tiene solamente y en promedio enseñanza primaria completa (7,6, 6,9 y 7,2 años, respectivamente). En el caso de los entrevistados la curtosis es leptocúrtica, su valor superior a o indica bastante concentración entorno a la escolarización media. Esto no ocurre en la generación parental donde hay mayor dispersión de datos entorno a la media, debido a un valor de curtosis menor a o (curtosis platicúrtica), aspecto reafirmado también por mayores valores en los coeficientes de variación. En Argentina, Chile, México y Perú (encuestas de hogares) encontraron que 30-50\% de los jóvenes que logran concluir sus estudios de nivel medio, acceden al nivel superior independiente del género. Asimismo se encontró que la estudiante típica del terciario constituye la primera generación de su familia que accede a la educación superior y en su mayoría trabaja al mismo tiempo que estudia (GARCÍA DE FANELLI, JACINTO; 2010).

Se debe agregar que Schultz (1961) desarrolló la Teoría del Capital Humano enfatizando en la educación como una inversión, donde el acceso a la educación y a la salud está determinado por los distintos ingresos, por ello sus consecuencias deberían ser tratadas como una forma de capital. Los avances en conocimientos y la mejora de la calidad de vida la población, son factores determinantes para el bienestar; es por esto que se deben buscar la adquisición de habilidades por medio de la escolarización e inversión sanitaria, en especial de las personas con bajos ingresos. Desde la óptica de este autor la educación, es considerada una inversión cuando

Rev.Cad.Comun. Santa Maria, v.21, n.3, art 6, p. 113 de 123, set/dez.2017 
se mejora el status social y económico, se adquieren bienes y servicios, es decir, cuando contribuye al bienestar humano.

Los resultados escolares se hallan determinados en altísima proporción por el factor familia (50-97\%) y por el efecto escuela en el resto. Con independencia de la magnitud atribuida a éste, la escuela importa, especialmente para los alumnos provenientes de un entorno familiar desaventajado. La mayor ganancia neta en términos de aprendizaje se produce entre los alumnos de más bajo nivel que estudian en una escuela efectiva y se hallan a cargo de un profesor efectivo. Sólo escuelas efectivas, con profesores efectivos, pueden compensar las desigualdades de origen familiar y así cumplir con el objetivo social de la educación (BRUNNER, ELAQUA; 2003). Sin embargo el fenómeno de estancamiento del crecimiento poblacional y la concentración urbana en ciudades reportado por Mazzei y De Souza (2012), pueden ser factores explicativos de la una búsqueda de más y mejores condiciones de educación. De hecho, el rendimiento escolar de los alumnos depende demasiado de los recursos culturales de sus padres para que la pura igualdad de oportunidades no sea una ficción. (DUBET, 2015). En el actual escenario en que se desenvuelven nuestras sociedades existen redes globales que articulan individuos, segmentos de población, regiones y ciudades, al mismo tiempo que excluyen otros tantos individuos, grupos sociales o territorios, tanto en lo económico, como en lo cultural y también como en lo social (VEIGA, 2004).

Por último, es posible decir que con un 0,95 de probabilidad o 95\% de confianza, el promedio de escolarización de los entrevistados y sus respectivas madres y padres y el promedio de ambos progenitores, se encuentran comprendidos en la media reportada en cada caso, mas o menos medio año de escolarización (0,41; 0,48; 0,62 y 0,44 años) (tabla 4). 


\begin{tabular}{|c|c|c|c|c|}
\hline Medidas & $\begin{array}{c}\text { Escola- } \\
\text { rización } \\
\text { (años) } \\
\text { entrevista- } \\
\text { do }\end{array}$ & $\begin{array}{c}\text { Escolariz- } \\
\text { ción } \\
\text { (años) } \\
\text { madre }\end{array}$ & $\begin{array}{c}\text { Escola- } \\
\text { rización } \\
\text { (años) } \\
\text { padre }\end{array}$ & $\begin{array}{c}\text { Escola- } \\
\text { rización } \\
\text { (años) } \\
\text { promedio } \\
\text { padres }\end{array}$ \\
\hline Promedio & 11,1 & 7,6 & 6,9 & 7,2 \\
\hline Mediana & 11 & 8 & 7 & 7 \\
\hline Moda & 11 & 8 & 11 & 6 \\
\hline Desvio estándar & 3,2 & 3,7 & 3,9 & 3,4 \\
\hline C.V.\% & 28,4 & 48,4 & 56,4 & 47,1 \\
\hline Error típico & 0,21 & 0,24 & 0,26 & 0,23 \\
\hline Curtosis & 0,83 & $-0,10$ & $-0,38$ & $-0,28$ \\
\hline $\begin{array}{c}\text { Coeficiente de } \\
\text { asimetría }\end{array}$ & $-0,54$ & 0,36 & 0,23 & 0,15 \\
\hline $\begin{array}{c}\text { Nivel confianza } \\
\text { (95,0\%) }\end{array}$ & 0,41 & 0,48 & 0,52 & 0,44 \\
\hline
\end{tabular}

Tabla 4. Medidas de tendencia central y de dispersión de la escolarización de los entrevistados y de sus progenitores.

Entre las variables que se han empleado para evaluar la correlación entre "capital cultural” y rendimiento educativo por género están la interacción de los progenitores con el entorno educativo de sus descendientes, la implicación de padres y madres en aprendizaje de sus hijos/as, aspiraciones del alumnado y otros (MARCENARO; LÓPES AGUDO, 2003). Los años de escolarización recibidos de los entrevistados (variable dependiente) encuentra una relación lineal y directa con el número de años de escolarización del promedio de los padres. Esta relación matemática muestra que una mayor educación de los padres explica una mayor educación en los hijos y viceversa (figura 3 ). 


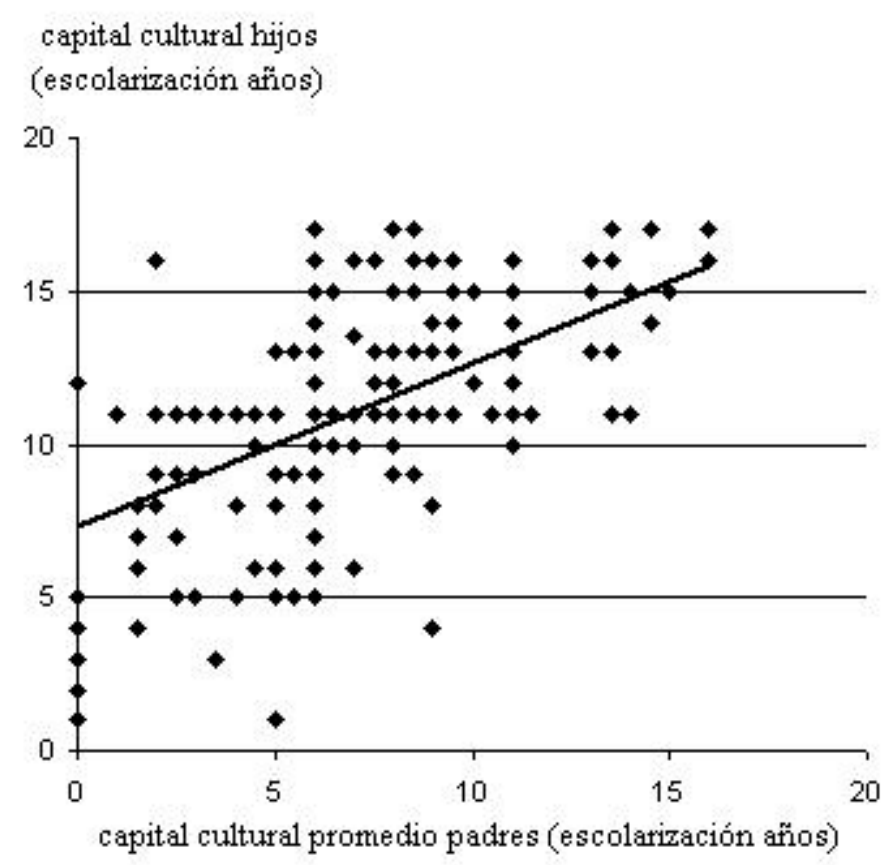

Figura 3. Correlación entre el capital cultural del promedio de los padres medido como número de años de escolarización en función del capital cultural de los hijos medido como número de años de escolarización de los entrevistados en la frontera uruguayo-brasilera.

En efecto, los coeficientes de correlación hijos-madres, hijos-padres e hijos-promedio padres reportaron valores de 0,52; 0,61 y 0,57 mostrando una relativa tendencia explicativa. Si se analizan estas tendencia bajo la perspectiva de modelo explicativo, se puede decir que la escolarización promedio de los padres explica un en 0,33 la escolarización de los hijos. Este valor no es bajo, pues a priori los individuos parten condicionados en un tercio por el nivel educativo de sus progenitores. Los otros dos tercios restantes estarían explicados por otras variables familiares y sociales de su entorno. Para llegar a niveles superiores de enseñanza, Charlot, (2005) menciona que la cuestión de las demandas formuladas por los estudiantes están relacionadas con el deseo, quien conduce al placer, al goce a través de un objeto, en este caso, la educación terciaria. Debemos tener presente que el sujeto se construye por la apropiación de un patrimonio humano, por la mediación de otro, y la historia del sujeto es también la de las formas de actividad que satisfacen el deseo, que producen placer y le dan sentido sustenta.

Resulta interesante resaltar que la escolarización del padre explica en mayor proporción el efecto sobre el hijo $(0,37)$, tiene mayor peso o pon- 
deración que la madre $(0,27)$ (tabla 5). No obstante, Fernández Aguerre (2002) remarca el efecto de la escolarización de la madre al mencionar que es costumbre señalar que en madres con un mínimo de algún año de educación terciaria o universitaria, habrán altas probabilidades de hallar hijos con código elaborado; y, por el contrario, en madres con una escolarización máxima de primaria es altamente probable encontrar un código restringido.

\begin{tabular}{|c|c|}
\hline Variables relacionadas & Valores \\
\hline $\begin{array}{c}\text { Correlación Pearson } \\
\text { hijos-madres }\end{array}$ & 0,52 \\
\hline $\begin{array}{c}\text { Correlación Pearson } \\
\text { hijos-padres }\end{array}$ & 0,61 \\
\hline $\begin{array}{c}\text { Correlación Pearson } \\
\text { hijos-promedio padres }\end{array}$ & 0,57 \\
\hline $\begin{array}{c}\text { Coeficiente determinación } \\
\text { hijos-madres }\end{array}$ & 0,27 \\
\hline $\begin{array}{c}\text { Coeficiente determinación } \\
\text { hijos-padres }\end{array}$ & 0,33 \\
\hline $\begin{array}{c}\text { Coeficiente determinación } \\
\text { hijos-promedio padre }\end{array}$ & 0,37 \\
\hline
\end{tabular}

Tabla 5. Coeficientes de correlación y de determinación entre hijos y madres, hijos y padres e hijos y promedio de los padres.

De acuerdo con Xavier (2013) tradicionalmente se impuso un formato de familia nuclear, que se sedimentó en el modelo "bread winner" o de un solo aportante, que recayó en la figura del hombre a otros modelos que compitieron en el escenario de las relaciones de parentesco, hoy día han aparecido distintas formas de organización de la familia como diversas estrategias para enfrentar la realidad de la sociedad actual, sus conflictos, sus vicisitudes, sus barreras y sus activos. Entre los diversos factores que incidieron en la aparición y consolidación de estos nuevos arreglos familiares se encuentra el aumento de la tasa de divorcio, desdibujando la tradicional familia nuclear "breadwinner sistem", también las tasas de fecundidad por estrato social. Lo anterior está en consonancia con Cabella (2007) en cuanto a que en el transcurso de estas dos últimas décadas las características de la familia uruguaya experimentaron profundas transformaciones que obedecieron por un lado a la profundización del envejecimiento demográfico, que contribuyó a aumentar la importancia

Rev.Cad.Comun. Santa Maria, v.21, n.3, art 6, p. 117 de 123, set/dez.2017 
de los hogares unipersonales y de las parejas solas, y por otro, a los cambios vertiginosos en la formación y disolución de las uniones. Por último, la modalidad familiar nuclear tipo está en creciente competencia con una pluralidad de arreglos familiares. Con respecto a la tasa de divorcio, en el año 1975 era de 14.0 por cada 100 matrimonios (INE, 1996), mientras que en el año 2000 la tasa de divorcios creció a 49.1, esto significa que por cada dos matrimonios se produce un divorcio, lo que evidencia con claridad los profundos cambios que se han procesado y se continúa procesando en Uruguay (XAVIER, 2013).

Asociado a la calidad de la educación y a baja motivación, Bauman (2007) afirma que el "síndrome de la impaciencia" trasmite el mensaje en que el tiempo es un fastidio y una faena, una contrariedad, un desaire a la libertad humana a los derechos humanos. Esperar se ha convertido en una circunstancia intolerable los profesores solo piensan en jubilarse y que su actividad en la educación acabe rápido sin ningún tipo de compromiso. Es interesante marcar que el despoblamiento de la faja fronteriza es más acentuado para el género masculino, pues Mazzei y De Souza (2012) mencionan que entre las ciudades brasileñas en un contexto mayor de decrecimiento poblacional de sus respectivos municipios, únicamente crece la ciudad de Chui y la que más decrece es la ciudad de Livramento. Las ciudades uruguayo-brasileras coinciden con la mayor proporción de mujeres registrada en toda el área binacional y Rivera es la ciudad que muestra un mayor valor en esa relación.

\section{CONSIDERACIONES FINALES}

a) El espacio fronterizo está diferenciado del territorio político al que pertenece, es una interfase con identidad propia que de acuerdo a cifras oficiales, presenta un menor desarrollo humano en todos sus municipios en comparación con las capitales de estado. En lo referente a la dimensión educativa del desarrollo humano, las políticas de descentralización no logran disminuir la equidad en comparación con los territorios con mayor incidencia de las respectivas ciudades metrópolis.

b) Este trabajo concuerda con lo sustentado por la teoría, pero en este caso el efecto ha sido mensurado, hallándose que la es-

Rev.Cad.Comun. Santa Maria, v.21, n.3, art 6, p. 118 de 123, set/dez.2017 
colarización de los hijos está condicionada por lo menos en una tercera parte por el nivel de escolarización de sus padres en lo que se puede señalar herencia del capital cultural vinculado a los años de escolarización del entorno familiar próximo. Los otros dos tercios estarían explicados por otras variables familiares de su entorno, capacidades del sujeto y contexto social de enclave.

c) La metodología desarrollada puede ser ampliada y aplicada en otros municipios, seria conveniente incluir información económica de los entrevistados para tener una visión más global del fenómeno social. 
CADERNOS DE COMUNICAÇÃO

UNIVERSIDADE FEDERAL DE SANTA MARIA

\section{REFERENCIAS}

ANEP. 2000. Evaluaciones nacionales en primaria 1995-1999. https://www.ineed.edu. uy/images/pdf/aristas/anep_2000_evaluaciones-de-aprendizajes-primaria-1995-1999. pdf. Acesso em 16 de Fevereiro de 2017.

ATLAS BRASIL. Atlas do desenvolvimento humano no Brasil. Índice de desarrollo humano - Brasil - RS por municipios. http://www.atlasbrasil.org.br/2013/ranking. Acesso em 16 de Fevereiro de 2017.

BAUMAN, Z. Los retos de la educación en la modernidad líquida. E. Gedisa, España. 2007

CHARLOT, B. La relación con el saber, formación de maestros y profesores, educación y globalización. Ed. Trilce. Montevideo. 2008.

BOURDIEU, P. El sentido práctico. Taurus; Madrid, 1993.

BOURDIEU, P. La distinción. Criterio y bases sociales del gusto, Madrid: Taurus. 1988.

BOURDIEU, P. Poder, derecho y clases sociales, Bilbao: Desclée de Brouwer. 2000.

BOURDIEU, P. Capital cultural, escuela y espacio social. Argentina: Siglo XXI, 2010.

BRASIL. Lei n 13.005 de 25 de junho de 2014. http://www.planalto.gov.br/ccivil_03/_ Ato2011-2014/2014/Lei/L13005.htm. Acesso em 16 de Dezembro de 2015

BRUNNER, J.; G. ELACQUA. Entre la desigualdad y la efectividad, Capital humano en Chile. Informe capital humano en Chile. Universidad Adolfo Ibañez, Chile 159 p. 2003. http://www.oei.es/historico/etp/informe_capital_humano_chile_brunner.pdf. Acesso em 15 de Fevereiro de 2017.

CALHOUN, C., LIPUMA, E. y POSTONE, M. (Ed.). Bourdieu: Critical Perspectives. Polity Press; Oxford. 1993.

FALCO PAULA GLÁUCIA DE, SOARES JÚNIOR FJ. e GONÇALVES ALTAF J. 2015. Avaliação da Demanda por Novos Cursos de Ensino Superior na Cidade de Juiz de Fora. www.aedb.br/seget/lista_artigos_aprovados.pdf. Acesso em $1^{\circ}$ de Dezembro de 2015.

Rev.Cad.Comun. Santa Maria, v.21, n.3, art 6, p. 120 de 123, set/dez.2017 
FERNÁNDEZ AGUERRE, T. Determinantes sociales e institucionales de la desigualdad educativa en sexto año de educación primaria de Argentina y Uruguay, 1999. Una aproximación mediante un modelo de regresión logística. Revista Mexicana de Investigación Educativa noviembre-diciembre. vol. 7, núm. 501-536. 2002.

GARCÍA DE FANELLI, A. JACINTO, C. . Equidad y educación superior en América Latina: El papel de las carreras terciarias y universitarias. Revista Iberoamericana de Educación superior. (1) (1). 58-75. 2010

INFANTE G. S.; ZÁRATE DE LARA G. P. Métodos estadísticos. Ed. Trillas. 646. México DF. 1994.

HANNERZ, U. Conexiones transnacionales. Cultura, gente, lugares. Madrid, Cátedra, 290 pp. 1998.

MARCENARO GUTIÉRREZ, O. LÓPEZ AGUDO, L.A. El efecto del capital cultural sobre el rendimiento educativo diferencial por género. IV Jornada Economía feminista. Universidad Pablo de Olavide, de Sevilla. 50 p. 2013. http://riemann.upo.es/personal-wp/ congreso-economia-feminista/files/2013/10/Marcenaro-Lopez.pdf. Acesso em 15 de Fevereiro de 2017.

MARCONI, M. A.; LAKATOS, E. Ma . Metodologia Científica. 5 ed. São Paulo: Atlas, 2011.

MARTÍN CRIADO, E. ; IZQUIERDO MARTÍN, Javier "Sociología de la gestión empresarial de la mano de obra", Sociología del Trabajo, nueva época, otoño-invierno, $\mathrm{n}^{\circ} 17$. 1992.

MOURÃO-JUNIOR, C. A. Revista Interdisciplinar de Estudos Experimentais, vol. 1, n. 1, p. $26-28,2009$.

OLIVEROS, L. El concepto de frontera en el contexto y en la perspectiva de la integración andina, Documento de la CAN. 2002.

PNUD. Programa Naciones Unidas Desarrollo. 2016. Informe Regional sobre Desarrollo Humano para América Latina y el Caribe 2016. http://www.latinamerica.undp.org/ content/rblac/es/home/library/human_development/informe-regional-sobre-desarrollo-humano-para-america-latina-y-e/. Acesso em 16 de Fevereiro de 2017. 
PNUD. Programa Naciones Unidas Desarrollo . http://hdr.undp.org/es/content/el-\%C3\%ADndice-de-desarrollo-humano-idh. Acesso em 20 de Fevereiro de 2017.

ROESCH, S. M. A. Projetos de Estágio e de Pesquisa em Administração. 3 ed. São Paulo: Atlas, 2009.

RÓTULO, D. ; O. DAMIANI. El caso de la integración fronteriza Uruguay Brasil: dimensiones analíticas e hipótesis de trabajo preliminares. 2010. 37 p Universidad ORT Uruguay Fondo Internacional de Desarrollo Agrícola Naciones Unidas http://www.ort.edu. uy/facs/pdf/documentodeinvestigacion61.pdf. Acesso em 12 de Fevereiro de 2017.

TENTI FANFANI, E. Nuevos temas en la agenda de política educativa. Editorial Siglo XXI. Buenos Aires, 2008

TENTI FANFANI, E. La Escuela y la cuestión social. Ensayos de sociología de la educación. E.S. XXI. Argentina. 2011.

VALDÉS VENTO, AC; I del R. MORALES SUÁREZ; J. C. DÍAZ CABRERA; A.L. SÁNCHEZ FUENTES; R. CUALLAR ÁLVAREZ. Criterios de evaluación de la variable "pertinencia e impacto social" específicos para la carrera de Medicina. Educación Médica Superior. 2009; 23(3): 45-62. http://scielo.sld.cu/pdf/ems/v23n3/ems05309.pdf. Acesso em 22 de Fevereiro de 2017.

UNNE. Universidad Nacional del Nordeste, Argentina. Componentes del desarrollo humano. http://hum.unne.edu.ar/revistas/geoweb/Geo2/contenid/idhnea2.htm> Acesso em 20 de Fevereiro de 2017. 


\section{Ignacio Pablo Traversa Tejero}

Doutorado em Meio Ambiente e Desenvolvimento pela Universidade Federal de Pernambuco. Mestrado em Produtividade Florestal pelo Instituto Tecnológico Valle de Oaxaca, México. Professor efetivo adjunto da Universidade Federal do Pampa (UNIPAMPA) Campus Dom Pedrito.

E-mail: igtraversa@gmail.com

\section{Juan Francisco Xavier Alvez}

Tiene especialización en Argentina y Maestría en la Universidad Federal de Santa María, RS. Actúa como profesor efectivo del Consejo de Formación en Educación (CFE) de Uruguay. Profesor de Sociología. Investigador. Centro Regional de Profesores del Norte.

E-mail: jfxavieralvez@gmail.com

\section{Jorge Rodríguez Monteblanco}

Profesor Investigador. Tiene varias especializaciones en el área de la Educación. Actúa como profesor efectivo del Consejo de Formación en Educación (CFE) de Uruguay. E-mail: jrmonteblanco@gmail.com

RECEBIDO EM: 23/02/2017

ACEITO EM: 23/04/2017 